\title{
Pneumonia Characteristics of Hospitalized Children Infected with Macrolide-Resistant Mycoplasma Pneumoniae
}

\section{feifei cui}

Children's Hospital Capital Institute of Pediatrics

\section{Xiu-jun Tian}

Beijing Tropical Medicine Research Institute, Capital Medical University

Deli Xin ( $\nabla$ xind148@126.com)

Beijing Tropical Medicine Research Institute, Capital Medical University

\section{Xiao-hua Han}

Shengjing Hospital of China Medical University

\section{Liang-yu Wang}

Beijing Tropical Medicine Research Institute, Capital Medical University

\section{Hai-wei Dou}

Beijing Tropical Medicine Research Institute, Capital Medical University

\section{Ran Wei}

Beijing Tropical Medicine Research Institute, Capital Medical University

\section{Li-na Han}

Shengjing Hospital of China Medical University

Hui-sheng Yao

Shengjing Hospital of China Medical University

\section{Research Article}

Keywords: extra-pulmonary complications, clinical characteristics, Mycoplasma pneumoniae pneumonia, macrolide-resistant Mycoplasma pneumoniae

Posted Date: August 30th, 2021

DOl: https://doi.org/10.21203/rs.3.rs-675299/v1

License: (c) (i) This work is licensed under a Creative Commons Attribution 4.0 International License.

Read Full License 


\section{Abstract}

Background: To investigate the drug resistance and clinical characteristics of hospitalized children with drug-resistant Mycoplasma pneumoniae pneumonia (MRMP).

Methods: Sixty patients with MPP admitted to the Second Pediatric Respiratory Ward of Shengjing Hospital, Affiliated to China Medical University from November 2016 to February 2017 were enrolled in the study.

Results: Of these 53/60 (88.3\%) patients had Mycoplasma pneumoniae nucleic acid identified by throat swab. 23S rRNA $V$ region gene sequencing was performed, 47/49 (95.9\%) had mutation sites, including 46 cases of A2063G, one case of A2064G, two cases of no mutation, and a final drug resistance rate of $95.9 \%$. The summary characteristics of the 47 cases of drug-resistant MPP were based on 22 male and 25 female patients. The onset age was $6.9 \pm 2.5$ years and the total fever duration was $9.8 \pm 3.7$ days. The leukocyte count during the acute phase was $(8,300 \pm 4,200)$ cells $/ \mathrm{mm}^{3}, \mathrm{C}$-reactive Protein (CRP) was $18.2(8.2-32.5) \mathrm{mg} / \mathrm{L}$, neutrophil/lymphocyte ratio (NLR) was 2.1 (1.5-3.3), There was no significant difference between the acute phase and the convalescent phase for leukocyte count, $P=0.336$. The NLR and CRP levels were significantly higher during the acute phase compared to the recovery period $(P<$ 0.05). The level of lactate dehydrogenase (LDH) increased in $65.7 \%$ of patients, with a median of 248.5 (200.0-299.7) U/L. D-dimer levels were elevated in 59.4\% of patients, with a median of 301.0 (188.5$545.0) \mathrm{mg} / \mathrm{L}$. A total of $23 / 47$ (48.9\%) patients were diagnosed with severe MPP. The incidence of extrapulmonary complications was $38.2 \%$.

Conclusions: In summary, MRMP patients had a fever of long duration, higher inflammatory index, higher LDH and D-dimer levels, and an increased incidence of extra-pulmonary complications.

\section{Background}

Mycoplasma pneumoniae (MP) is the smallest prokaryote that can survive independently. It causes upper and lower respiratory tract infections, mostly in children and young people (Waites KB, 2009). A worldwide MP pandemic occurs every 3-7 years. Pneumonia caused by MP (MPP) is a common community-acquired pneumonia (CAP) in children, accounting for approximately $40 \%$ of CAP in children greater than 5 years of age (Rogozinski Le, 2017). Since MP does not contain a cell wall, it is naturally resistant to beta lactam antibiotics, which affect cell wall synthesis. Macrolides, tetracyclines, and fluoroquinolones are effective drugs against mycoplasma (Bradley JS, 2011). Since tetracycline drugs such as minocycline and doxycycline are only used in children greater than 8 years of age and since quinolones should be used with caution in children less than 18 years of age, macrolides are the first choice for MP infection in children.

However, recent MP resistance to macrolides has become a serious clinical problem. The rate of macrolide-resistant MP (MRMP) is as high as $87.7 \%$ (Guo DX, 2019). However, the clinical characteristics of drug-resistant MPP are rarely reported in China. In this study the drug resistance and clinical 
characteristics are summarized for 60 MPP children hospitalized in the Second Pediatric Respiratory Ward of Shengjing Hospital, Affiliated to China Medical University from 2016 to 2017.

\section{Methods}

\subsection{General information.}

1.1.1 Clinical data were assessed for 60 cases of MPP from the Second Pediatric Respiratory Ward of Shengjing Hospital, Affiliated to China Medical University from November 2016 to February 2017, which included 27 males and 33 females.

1.1.2 Inclusion criteria: (1) met the diagnostic criteria for MPP, had clinical symptoms such as fever, cough or wheezing, imaging showed infiltration shadow or large consolidation shadow in the lung, positive MP nucleic acid in pharyngeal swab and antibody titer for MP during the acute stage was greater than 1:160, or serum antibody titer increased or decreased at least four times or more when the acute state was compared to the recovery period, (2) age < 18 year, and (3) study inclusion approved by the ethics committee.

1.1.3 The diagnostic criteria for severe MPP were the latest domestic diagnostic criteria for severe CAP in children (Respiratory Group, 2013) including a diagnosis of MPP as well as: (1) poor general condition, (2) food refusal or dehydration, (3) disturbance of consciousness, (4) significant increase in respiratory rate (judgment criteria: RR of infants $\geq 70$ times / min, RR of older children $\geq 30$ times / min), (5) cyanosis, (6) dyspnea (groaning, flapping of nasal alar, three concave sign), (7) lung infiltration range: multi lobe involvement or 2 / 3 of a lung lobe, (8) pleural effusion, (9) pulse oxygen saturation $\leq 92 \%$, and (10) extra-pulmonary complications.

1.1.4 Exclusion criteria: (1) basic metabolic disorders, low immune function, or major chronic consumptive diseases and other basic diseases, (2) patients with previous recurrent wheezing or chronic cough were diagnosed as asthma, (3) premature infants, or (4) incomplete data.

\subsection{Observation index.}

Medical history collection included, (gender, age, duration of fever on admission, total heat duration, and complications), imaging examination, laboratory indicators (white blood cell count, serum C-reactive protein (CRP), neutrophil to lymphocyte ratio (NLR), lactate dehydrogenase (LDH), D-dimer, pathogenic indexes (nucleic acid detection, drug resistance gene detection, antibody titer), and treatment.

\subsection{Laboratory testing.}

Clinical specimens testing positive for MP were shipped to the Laboratory and tested by real-time PCR using a Universal Genomic DNA Kit (Beijing Kangwei Century Biotech Co., Ltd., China) for detection of MP as well as all point mutations in the 23S rRNA gene known to be associated with macrolide resistance. This analysis was based on the different melting points for the mutated nucleotide base pairs compared 
with those of wild type (Waites KB, 2017). Sanger sequencing using the same primers as for PCR was performed to confirm MP detection and the point mutations. Results were compared to MP M129 (GenBank accession no. X68422).

\subsection{Statistical analysis.}

Statistical analysis was performed using IBM SPSS software, version 16.0区SPSS Inc., Chicago, IL, USA for Windows. Continuous data were compared using Students't test. Differences in categorical variables were assessed with Yates' corrected Chi-square $(X)$ or Fisher's exact test as appropriate. To compare the laboratory data between the acute stage and convalescence, Student's T-test, Mann-Whitney U test, Chisquare test, and Fisher's exact test were used, as appropriate. $P$ values less than 0.05 were considered statistically significant.

\section{Results}

2.1 Basic data: 60 patients with MPP were assessed (27 males and 33 females), of which 53/60 (88.3\%) patients had Mycoplasma pneumoniae nucleic acid identified by throat swab. Forty-seven patients had macrolide resistant MPP (22 males and 25 females) and two patients had no mutation as judged by $23 \mathrm{~S}$ rRNA $V$ region gene sequencing. Of the 47 patients, the onset age was $6.9 \pm 2.5$ years, with total fever duration $9.8 \pm 3.7$ days, and cough relief time $13.1 \pm 3.9$ days. During the acute phase, leukocyte count was $(8,300 \pm 4,200)$ cells $/ \mathrm{mm}^{3}$, CRP was $18.2(8.2-32.5) \mathrm{mg} / \mathrm{L}$, and neutrophil/lymphocyte ratio was 2.1 (1.5-3.3). LDH increased in 65.7\% of the patients, with a median of 248.5 (200.0-299.7) U/L, D-dimer levels increased in $59.4 \%$ of patients with a median of $301.0(188.5-545.0) \mathrm{mg} / \mathrm{L}, 30 / 47(63.8 \%)$ had lung consolidation, $13 / 47$ (27.6\%) had double lung involvement, and 23/47 (48.9\%) were diagnosed as severe MPP. The total incidence of extra-pulmonary complications was $38.2 \%$. Among those, $8 / 47$ (17\%) were complicated with pleural effusion, 8/47 (17\%) had central nervous system involvement, and 2/47 (4.2\%) had functional liver damage. Treatment: 13/47 (27.6\%) patients were treated with glucocorticoid, $5 / 47(10.6 \%)$ were treated with human serum immunoglobulin, and $7 / 47(14.9 \%)$ were treated with fiberoptic bronchoscopy. See Table 1.

\section{Table 1. Characteristics of hospitalized patients with MRMP.}




\begin{tabular}{|c|c|}
\hline Index & $\operatorname{MRMP}(n=47)$ \\
\hline Age, years & $6.9 \pm 2.5$ \\
\hline Gender \Male/Female $\rrbracket$ & $22 / 25$ \\
\hline Duration of fever at admission, days & $7.4 \pm 3.2$ \\
\hline Total duration of fever, days & $9.8 \pm 3.7$ \\
\hline Cough relief time, days & $13.1 \pm 3.9$ \\
\hline Leukocyte (cells/mm³) & $8,300 \pm 4,200$ \\
\hline NLR & $2.1 \otimes 1.5-3.3 \otimes$ \\
\hline CRP®mg/L) & $18.2 \varangle 8.2-32.5 \rrbracket$ \\
\hline *LDH囚U/L囚 & 248.5®200.0-299.7凶 \\
\hline **D-Dimer囚UG/L】 & 301.0ه188.5-545.0ه \\
\hline \multicolumn{2}{|l|}{ Radiographic findings } \\
\hline Bilateral infiltration, $\mathrm{n} \otimes \% \rrbracket$ & $13 \rrbracket 27.6 \rrbracket$ \\
\hline Lobar Consolidation, $\mathrm{n} \otimes \% \square$ & $30 \rrbracket 63.8 \rrbracket$ \\
\hline \multicolumn{2}{|l|}{ Total complication, $\mathrm{n} \rrbracket \% \rrbracket$} \\
\hline Pleural effusion, $n \rrbracket \% \rrbracket$ & $8 \otimes 17.0 \otimes$ \\
\hline Central nervous system involvement, $\mathrm{n} \otimes \% \rrbracket$ & $8 \otimes 17.0 \rrbracket$ \\
\hline Liver function impairment, $n \rrbracket \% \rrbracket$ & $2 \varangle 4.2 \rrbracket$ \\
\hline \multicolumn{2}{|l|}{ Other interventions } \\
\hline Glucocorticoid, $n \rrbracket \% \bigotimes$ & $13 \varangle 27.6 \rrbracket$ \\
\hline Immunoglobulin, $n \rrbracket \% \bigotimes$ & $5 \rrbracket 10.6 \rrbracket$ \\
\hline Fibrobronchoscopy, n囚\%区 & $7 \rrbracket 14.9 \rrbracket$ \\
\hline \multicolumn{2}{|l|}{ Combined use of cephalosporins } \\
\hline$n=0 \otimes \% \rrbracket$ & $1 \otimes 2.1 \rrbracket$ \\
\hline$n=1 \rrbracket \% \rrbracket$ & $26 \rrbracket 55.3 \rrbracket$ \\
\hline$N=2 \rrbracket \% \bigotimes$ & $16 \rrbracket 34.0 \rrbracket$ \\
\hline $\mathrm{n} \geq 3 \rrbracket \% \otimes$ & $4 \rrbracket 8.5 \rrbracket$ \\
\hline
\end{tabular}

Data are presented as number (percentage), median $\left(25^{\text {th }}-75^{\text {th }}\right.$ percentile), mean \pm SD . 
*The normal range of $\mathrm{LDH}$ is $103-227 \mathrm{U} / \mathrm{L}$, * * the normal range of $\mathrm{D}$-dimer is $0-52 \mathrm{UG} / \mathrm{L}$.

2.2 MP detection: during the acute stage the positivity rate for throat swab MP nucleic acid was $53 / 60$ $\nabla 88.3 \%), 35 / 60$ cases $(58.3 \%$ ) had mycoplasma antibody titers $>1: 160$ during the acute stage, during the recovery stage the antibody titer for MP in 60 cases was higher than 1:160 or four times higher than during the acute stage.

2.3 Detection of macrolide resistant sites: four of 53 pharyngeal swabs failed to detect 23S rRNA V mutation, mutation sites were found in the remaining $47 / 49$, the macrolide-resistance rate was $95.9 \%$ including 46 cases of the A2063G mutation, one case of the A2064G mutation, and two cases of no detected mutation.

2.4 NLR and CRP: during the acute phase the number of leukocytes was higher than normal in $30 \%$ of patients with no significant difference between the acute and convalescent stages $(P=0.336)$, levels of NLR and CRP during the acute phase were significantly higher than those during the convalescent stage $(P<0.05)$. See Table 2.

\section{Table 2. Comparison of Leukocyte, NLR, and CRP between acute phase and convalescent stage in hospitalized patients with MRMP.}

\begin{tabular}{|c|c|c|c|}
\hline & acute phase & convalescence stage & *P-value \\
\hline Leukocyte (cells $/ \mathrm{mm}^{3}$ ) & $8,300 \pm 4,200$ & $7,200 \pm 2,200$ & 0.336 \\
\hline NLR & $2.1 \otimes 1.5-3.3 \rrbracket$ & 1.1凶0.8-1.7区 & 0.000 \\
\hline $\mathrm{CRP} \bowtie \mathrm{mg} / \mathrm{L})$ & $18.2 \llbracket 8.2-32.5 \rrbracket$ & $3.1 \rrbracket 3.1-3.4 \rrbracket$ & 0.000 \\
\hline
\end{tabular}

Data are presented as median $\left(25^{\text {th }}-75^{\text {th }}\right.$ percentile $)$, mean \pm SD.

*There was no significant difference in Leukocytes between the acute phase and convalescent stage, $\mathrm{P}=$ 0.336 , the levels of NLR and CRP during the acute phase were significantly higher than those during the convalescent stage, $\mathrm{P}<0.05$.

\section{Discussion}

Okazaki, a Japanese scholar, first discovered MRMP in 2000. The antibiotic resistance was found to be related to a point mutation in the 23 S rRNA gene (Okazaki N, 2001). Since then, investigation of MRMP has intensified. Zhao Fei et al. (Zhao F, 2013, Zhao F, 2019, Zhao F, 2019) monitored MRMP in six Chinese cities from 2008 to 2018 and found the macrolide-resistance rate to be as high as $65 \%-98 \%$. The MRMP rate in Japan was $81.6 \%$ in 2008 and $43.6 \%$ in 2015. Examples of resistance rates are, $80 \%$ in Korea in 2015, 13.2\% in the United States in 2013, 8.3\% in France in 2011, and 3.1\% (Takaaki Tanaka,2017, Lee, E, 2017, Zheng X, 2015, Pereyre S, 2013, Dumke R, 2015) in Germany in 2011-2012. Overall, the macrolideresistance rate in Asian countries is much higher than that in European and American countries. Analysis 
of the clinical data for MPP children hospitalized in the Second Pediatric Respiratory Ward of Shengjing Hospital, Affiliated to China Medical University from November 2016 to February 2017 found the resistance rate of MP to be as high as $95.9 \%$. Only one case had the A2064G mutation, with the remainder the classic mutation site, A2063G. These data demonstrate the prevalence of MRMP in hospitalized Chinese children.

For the 47 hospitalized children with MRMP pneumonia, the average fever duration was more than 9 days, which is longer than that of non-macrolide-resistant MPP. Fever was approximately $8.0 \pm 6.0$ days $(n=12)$ (Eun LeeđKorean J Pediatr 2017) and $6.0 \pm 3.5$ days $(n=96)$ (Kim YJ囚J Korean Med Sci 2017) for non-macrolide-resistant MPP. These observations demonstrate MRMP pneumonia to have longer fever duration, which is consistent with this study. The incidence of leukocytosis was approximately $30 \%$, which is consistent with previous reports (Stevens, D. 1978, Medjo B, 2014). Those reports found approximately $27.6 \%$ of cases to involve bilateral lungs, which was higher than the $20 \%$ of cases in a previous report (Ferwerda, A, 2001). Extra-pulmonary complications were as high as $38.3 \%$, which was higher than the 17\% reported previously (Yang TI, 2019). Herein, CRP and NLR during the acute phase were significantly higher than those in the recovery stage. CRP is an acute phase protein secreted by the liver in response to a variety of inflammatory cytokines. NLR can accurately predict the severity of and prognosis for CAP, as well as assist in risk determination for severe pneumonia patients with complications (Che-Morales JL, 2019). Increases in CRP and NLR reflect aggravation of the inflammatory response, suggesting a more serious disease. Approximately half of the cases within the macrolideresistant group had severe pneumonia. For more than half of the cases, LDH and D-dimer levels were higher than the normal value, suggesting that MRMP likely involves not just the lungs but also other organs. Myocardial, liver, pancreas, and other injuries result in elevated LDH. Hypoxia and endotoxin stimulate inflammatory cells to release a variety of inflammatory mediators that result in vascular endothelial cell damage and coagulation events that significantly increase plasma D-dimer (Yuan SJ, 2017). D-dimer levels can be used as one of the critical markers for monitoring inflammation and severe infection (Wu HB, 2017). Chen Yu (Chen Yu, 2018) found that LDH was higher in patients with MRMP compared with patients with non-drug resistant MPP. Ryou Kawamata concluded that the higher the LDH, the more severe the MPP (Kawamata R, 2015). These results suggest that LDH and D-dimer levels are high in more than half of the cases with MRMP pneumonia cases, indicating that the pneumonia caused by MRMP is more serious clinically.

The duration of fever induced by MRMP is longer than that induced by non- macrolide-resistant MP (Suzuki S, 2006, Zhou YL, 2014, Yao HS, 2016, Yang TI, 2019). Further, fever duration is longer after administration of macrolides, hospitalization time is longer, and extra-pulmonary complications are more serious. MRMP infections are more common in immune suppressed individuals and in cancer patients. The predominant clinical difference between MRMP and non- macrolide-resistant MP is the duration of fever, with no significant difference in lung imaging, laboratory measures, or extra-pulmonary complications (Deng H, 2018, Wu HM, 2013, KB Waites, 2019). According to Jae Hong Choi, MRMP involves the lobes of the lungs without other clinical or laboratory signs of infection (Choi JH, 2019). AE Yoon et al. (Yoon IA, 2017) showed that the duration of fever was related to the severity of lung imaging, 
but not to drug resistance. These inconsistent reports may be due to differences in sample size or treatment methods.

In this study, the macrolide-resistance rate of MPP inpatients was as high as $95.9 \%$, which was significantly higher than the concomitant rate of $65.4 \%$ for outpatients (Zhao F, 2019). We infer that MRMP infection is more likely to cause refractory/severe MPP that results in a higher treatment failure rate for outpatients, such that the macrolide-resistance rate for hospitalized patients is significantly increased. The results of this study identify the total fever duration of drug-resistant MPP patients is 9 days, which is significantly longer than the $5-6$ days for patients infected with non-macrolide-resistant MP (Medjo B, 2014). These patients were treated with glucocorticoid $(n=13)$ and immunoglobulin $(n=5)$ to shorten the course of fever. Approximately half of macrolide-resistant MPP develops into severe MP. The rate of extra-pulmonary complications is high, with lungs seriously affected as judged by imaging. Therefore, MRMP is more likely to develop into a severe case. We also found severe MPP cases in patients with non-macrolide-resistant MP. Severe MPP is not only related to the macrolide-resistance of the pathogen but also to bacterial load, bacterial virulence, and the immune status of the patient. Future investigations need to consider the influence of these other factors on severe MPP outcomes.

Limitations of this study: This study summarizes the drug resistance and case characteristics of hospitalized patients, but does not include analysis of outpatients with mycoplasma pneumonia. This short term study was performed at a single center and only assessed drug resistant MPP in the Pediatric Department of Shengjing Hospital in the winter of 2016. Further, there were few non-drug resistant cases in this study and comparisons between groups is therefore limited. The results of this study need to be confirmed with a larger sample size and a more in-depth study.

Conclusions: The majority of MPP patients were found to be infected with macrolide-resistant MP (95.9\%). The children with MRMP pneumonia were mainly school-aged with a mean age of 6.9 years. Average total fever duration was more than 9 days. Leukocyte count during the acute phase was slightly increased in approximately $30 \%$ of cases. CRP and NLR during the acute phase were significantly higher than during the recovery period. LDH and D-dimer levels were increased in more than half of the patients with MRMP. The overall incidence of extra-pulmonary complications was $38.2 \%$. Therefore, MRMP patients exhibited longer fever duration, higher inflammatory index, higher levels of LDH and D-dimer, increased incidence of extra-pulmonary complications, and were likely to develop severe pneumonia. MP resistance requires strict attention, with appropriate administration of antibiotics, and early identification of severe cases. Early glucocorticoid intervention can prevent and/or reduce complications and improve prognosis.

\section{Abbreviations}

MPP- Mycoplasma pneumoniae pneumonia

CRP- C-reactive Protein 
NLR- neutrophil/lymphocyte ratio

LDH- lactate dehydrogenase

MRMP- macrolide-resistant Mycoplasma pneumoniae pneumonia

\section{Declarations}

Conflict of Interest Statement: The authors declared no potential conflicts of interest with respect to the research, authorship or publication of this article.

Acknowledgement $\varangle$ We thank International Science Editing for editing this manuscript. (http://www.internationalscienceediting.com )

\section{References}

1. Waites KB, Atkinson TP. The role of Mycoplasma in upper respiratory infections. Curr Infect Dis Rep. 2009 May,11(3):198-206.

2. Rogozinski Le, Alverson BK, Biondi EA. Diagnosis and treatment of Mycoplasma pneumoniae in children.Minerva Pediatr. 2017 Apr,69(2):156-160. doi: 10.23736/S0026-4946.16.04866-0.

3. Bradley JS, Byington CL, Shah SS, Alverson B, Carter ER, Harrison C, et al. The management of community-acquired pneumonia in infants and children older than 3 months of age: clinical practice guidelines by the Pediatric Infectious Diseases Society and the Infectious Diseases Society of America. Pediatric Infectious Diseases Society and the Infectious Diseases Society of America. Clin Infect Dis.2011,53:e25-e76.

4. Guo DX, Hu WJ, Wei R, Wang H, Xu BP, Zhou W, et al. Epidemiology and mechanism of drug resistance of Mycoplasma pneumoniae in Beijing, China: A multicenter study. Bosn J Basic Med Sci. 2019 Aug, 19(3): 288-296. doi: 10.17305/bjbms.2019.4053.

5. Respiratory group, society of Pediatrics, Chinese Medical Association. Guidelines for the management of community acquired pneumonia in children (2). Chin J Pediatr, November 2013, Vol. 51, No. 11: 856-862.

6. Waites KB., Xiao L, Liu Y, Balish MF, Atkinson TP. Mycoplasma pneumoniae from the Respiratory Tract and Beyond.Clin Microbiol Rev. 2017 Jul, 30(3): 747-809. Published online 2017 May 24. doi: 10.1128/CMR.00114-16.

7. Okazaki N, Narita M, Yamada S, Izumikawa K, Umetsu M, Kenri T, et al. Characteristics of macrolideresistant Mycoplasma pneumoniae strains isolated from patients and induced with erythromycin in vitro. Microbiol Immunol 2001,45:617-20.

8. Zhao F, Liu G, Wu J. Cao B, Tao XX, He LH, et al. Surveillance of Macrolide-Resistant Mycoplasma pneumoniae in Beijing, China, from 2008 to 2012. . Antimicrobial Agents and Chemotherapy. March 2013 Volume 57 Number 3:1521-1523. 
9. Zhao F, Liu JR, Shi WX, Huang F, Liu LY, Zhao SY, et al. Antimicrobial susceptibility and genotyping of Mycoplasma pneumoniae isolates in Beijing, China, from 2014 to 2016. Antimicrobial Resistance and Infection Control (2019) 8:18. doi.org/10.1186/s13756-019-0469-7.

10. Zhao F, Li J, Liu JR, Guan XM, Gong J, Liu LY, et al. Antimicrobial susceptibility and molecular characteristics of Mycoplasma pneumoniae isolates across different regions of China. Antimicrob Resist Infect Control. 2019, 8: 143. doi: 10.1186/s13756-019-0576-5.

11. Tanaka T, Oishi T, Miyata I, Wakabayashi S, Kono M, Ono S, et al. Macrolide-Resistant Mycoplasma pneumoniae Infection, Japan, 2008-2015. Emerg Infect Dis. 2017 Oct, 23(10): 1703-1706.doi: 10.3201/eid2310.170106.

12. Lee E, Cho H.J, Hong SJ, Lee J, Sung H, Yu J. Prevalence and clinical manifestations of macrolide resistant Mycoplasma pneumoniae pneumonia in Korean children. Korean J. Pediatr. 2017, 60, 151157.

13. Zheng X, Lee S, Selvarangan R, Qin X, Tang YW, Stiles J, et al. Macrolide-Resistant Mycoplasma pneumoniae, United States. Emerg Infect Dis. 2015, 21:1470-2. 10.3201/eid2108.150273.

14. Pereyre S, Touati A, Petitjean-Lecherbonnier J, Charron A, Vabret A, Bébéar $C$. The increased incidence of Mycoplasma pneumoniae in France in 2011 was polyclonal, mainly involving M. pneumoniae type 1 strains. Clin Microbiol Infect. 2013,19: E212-7. doi: 10.1111/1469-0691.12107.

15. Dumke R, Schnee C, Pletz MW, Rupp J, Jacobs E, Sachse K, et al. Mycoplasma pneumoniae and Chlamydia spp. infection in community-acquired pneumonia, Germany, 2011-2012. Emerg Infect Dis. 2015, 21:426-34. doi: 10.3201/eid2103.140927.

16. Lee E, Cho HJ , Hong SJ, Lee J, Sung H, Yu JH. Prevalence and clinical manifestations of macrolide resistant Mycoplasma pneumoniae pneumonia in Korean children. Korean J Pediatr. 2017 May, 60(5): 151-157. doi: 10.3345/kjp.2017.60.5.151.

17. Kim YJ, Shin KS, Lee KH, Kim YR, Choi JH. Clinical Characteristics of Macrolide-Resistant Mycoplasma pneumoniae from Children in Jeju. J Korean Med Sci. 2017 Oct, 32(10):1642-1646. doi: 10.3346/jkms.2017.32.10.1642.

18. Stevens D, Swift PG, Johnston PG, Kearney PJ, Corner BD, Burman D. Mycoplasma pneumoniae infections in children. Arch Dis Child. 1978 Jan, 53(1):38-42. doi: 10.1136/adc.53.1.38.

19. Medjo B, Atanaskovic-Markovic M, Radic S, Nikolic D, Lukac M, Djukic S. Mycoplasma pneumoniae as a causative agent of community-acquired pneumonia in children: clinical features and laboratory diagnosis. Ital J Pediatr. 2014 Dec 18,40:104. doi: 10.1186/s13052-014-0104-4.

20. Ferwerda A, Moll HA, Groot R. Respiratory tract infections by Mycoplasma pneumoniae in children: a review of diagnostic and therapeutic measures. Eur J Pediatr. 2001 Aug,160(8):483-91. doi: $10.1007 / \mathrm{s} 004310100775$.

21. Yang TI, Chang TH, Lu CY, Chen JM, Lee PI, Huang LM, et al. Mycoplasma pneumoniae in pediatric patients: Do macrolide-resistance and/or delayed treatment matter? Journal of Microbiology, Immunology and Infection, 2019, 52(2):329-335. doi: 10.1016/j.jmii.2018.09.009. 
22. Che-Morales JL , Cortes-Telles A. Neutrophil-to-lymphocyte Ratio as a Serum Biomarker Associated with Community Acquired Pneumonia. Rev Med Inst Mex Seguro Soc. 2019 Mar 15,56(6):537-543.

23. Yuan SJ, Xie HT and Li ZL. Clinical significance of hypersensitive C-reactive protein, fribrinogen and D-dimmer in connective tissue disease-related interstitial lung disease. Nan Fang Yi Ke Da Xue Xue Bao 37: 415-419, 2017 (In Chinese). doi: 10.3969/j.issn.1673-4254.2017.03.25.

24. Wu HB, Ma WG, Zhao HL, Zheng J, Li JR, Liu O, et al. Risk factors for continuous renal replacement therapy after surgical repair of type A aortic dissection. J Thorac Dis. 2017 Apr,9(4):1126-1132. doi: 10.21037/jtd.2017.03.128.

25. Chen Yu, Tian WM, Chen Q, Zhao HY, Huang P, Lin ZQ, et al. Clinical Features and Treatment of Macrolide-Resistant Mycoplasma Pneumoniae Pneumonia in Children. Zhongguo Dang Dai Er Ke Za Zhi. 2018 Aug,20(8):629-634. doi: 10.7499/j.issn.1008-8830.2018.08.006.

26. Kawamata R, Yokoyama K, Sato M, Goto M, Nozaki Y, Takagi T, et al. Utility of Serum Ferritin and Lactate Dehydrogenase as Surrogate Markers for Steroid Therapy for Mycoplasma Pneumoniae Pneumonia. J Infect Chemother. 2015 Nov,21(11):783-9. doi: 10.1016/j.jiac.2015.07.009.

27. Suzuki S, Yamazaki T, Narita M, Okazaki N, Suzuki I, Andoh T, et al. Clinical Evaluation of MacrolideResistant Mycoplasma pneumoniae. Antimicrob Agents Chemother. 2006 Feb,50(2):709-12.doi: 10.1128/AAC.50.2.709-712.2006.

28. Zhou YL, Zhang YY, Sheng YJ, Zhang L, Shen Z, et al. More Complications Occur in MacrolideResistant than in Macrolide- sensitive Mycoplasma pneumoniae Pneumonia. Antimicrob Agents Chemother. 2014 Feb, 58(2): 1034-1038. doi: 10.1128/AAC.01806-13.

29. Yao HS, Zhang R, Liu LY, Wang J, Yi LL, Han XH. Correlation of Mycoplasma pneumoniae resistance gene detection and refractory Mycoplasma pneumoniae pneumonia. Int J Pediatr, Jun 2016, Vol.43, N0.6:492-496. doi:10.3760/cma.J.issn.1673-4408.2016.06.017.

30. Deng H, Rui J, Zhao DY, Liu F. Mycoplasma Pneumoniae 23S rRNA A2063G Mutation Does Not Influence Chest Radiography Features in Children With Pneumonia. J Int Med Res. 2018 Jan,46(1):150-157. doi: 10.1177/0300060517716312.

31. Wu HM, Wong KS, Huang YC, Lai SH, Tsao KC, et al. Macrolide-resistant Mycoplasma Pneumoniae in Children in Taiwan. J Infect Chemother. 2013 Aug, 19(4):782-6. doi: 10.1007/s10156-012-0523-3.

32. Waites KB, Ratliff A, Crabb DM, Xiao L, Qin X, Selvarangan R, et al. Macrolide-Resistant Mycoplasma Pneumoniae in the United States as Determined From a National Surveillance Program. J Clin Microbiol. 2019 Oct 3,57(11): e00968-19. doi: 10.1128/JCM.00968-19.

33. Choi JH, Seong GM, Ko Y, Kim YR, Kim C. Prevalence and Clinical Features of CommunityAcquired Pneumonia Caused by Macrolide-Resistant Mycoplasma pneumoniae Isolated From Adults in Jeju Island. Mircob Drug Resist. 2019 May,25(4):577-581. doi: 10.1089/mdr.2018.0295.

34. Yoon IA, Hong KB, Lee HJ, Yun KW, Park JY, et al. Radiologic Findings as a Determinant and No Effect of Macrolide Resistance on Clinical Course of Mycoplasma Pneumoniae Pneumonia. BMC Infect Dis. 2017 Jun 7,17(1):402. doi: 10.1186/s12879-017-2500-z. 